Article

\title{
The Can-Order Policy for One-Warehouse N-Retailer Inventory System: A Heuristic Approach
}

\author{
Varaporn Pukcarnon, Paveena Chaovalitwongse*, and Naragain Phumchusri \\ Department of Industrial Engineering, Faculty of Engineering, Chulalongkorn University, Bangkok 10330, \\ Thailand \\ *E-mail: Paveena.c@chula.ac.th
}

\begin{abstract}
We study an application of the can-order policy in one-warehouse n-retailer inventory systems, and propose a heuristic approach for setting the appropriate inventory policy. On the can-order policy, an order is triggered when a retailer's inventory position reaches its must-order level. Then other retailers are examined whether their inventory reaches their can-order level, and if so they are filled by this order as well. Warehouse fulfills all involved retailers' inventory to their order-up-to levels. The can-order policy is not only able to save the total system-wide cost from joint replenishment, but it is also simple to use. Computer simulation is utilized to preliminarily study and to determine the best-known solution. We propose a heuristic approach utilizing the decomposition technique, iterative procedure, and golden section search to obtain the satisfying total system-wide cost. This can save our computational time to find the appropriate inventory policy setting from the reduced search space. We found that the proposed heuristic approach performs very well with the average cost gap of less than $2 \%$ comparing to the best-known solution. Thus, the can-order policy can be very useful for such systems.
\end{abstract}

Keywords: Can-order policy, joint replenishment, one-warehouse n-retailer inventory system, heuristic approach.

ENGINEERING JOURNAL Volume 18 Issue 4

Received 27 September 2013

Accepted 25 March 2014

Published 16 October 2014

Online at http://www.engj.org/

DOI:10.4186/ej.2014.18.4.53 


\section{Introduction}

This paper focuses on the one-warehouse n-retailer inventory system (OWNR) which is a general pattern of two-echelon supply chain. Such system confronts the uncertainty of demand in reality. Supply coordination called "centralized control" has been widely applied to reduce the total system-wide cost and the demand variation, as well as to improve supply performance including inventory planning process [1-3]. In addition, various supply chains give their attention into continuous replenishment according to the responsive information technology. This can not only reduce their buffer stocks but also improve the entire system's performance. Hence, we concentrate on the inventory policy for controlling such system under stochastic demand and continuous replenishment.

A number of researches on OWNR have been conducted under either continuous or periodic replenishment. They proposed mathematical models and solution approaches for setting an appropriate inventory policy. Most of the works studied two major types of the inventory policies: Fixed-interval orderup-to policies and Stock-based batch-ordering policies, on different conditions and relevant parameters. Further details can be seen in the reviews of Axsäter et al. [4], Wang et al. [5], and Schneider et al. [6]. Focusing on continuous replenishment, most researches manage multiple retailers by individual ordering decision. Factually, multiple retailers can coordinate their ordering decision to share the ordering cost when an order is triggered. It creates an opportunity for reducing the total system-wide cost. We found that just a few works concerned this cost-saving opportunity in their ordering decisions.

With regard to coordinated ordering decision, most literatures applied joint replenishment problem (JRP) to OWNR due to the similarity of cost functions and solution procedures [7, 8]. JRP is originally developed for the multi-product inventory problem with the replenishment coordination of a group of items jointly ordered from the same supplier. Cheung and Lee [9] studied the $(Q, S)$ policy. When the cumulative demands over all retailers reach a given $Q$ units (i.e. truckload size for all retailers in single trip), an order is placed at the warehouse to replenish the retailer to their order-up-to level $S$. At the warehouse, a traditional reorder point-fixed order quantity policy was employed.

Özkaya [10] proposed analytical models and heuristic approaches for four types of joint replenishment policy at the retailers, and utilized a traditional reorder point-based stock policy at the warehouse. Four types of joint replenishment policy are $(Q, S)$ policy, $(Q, S, T)$ policy, $(Q, S \mid T)$ policy, and $(s, S-1, S)$ policy. The $(Q, S)$ policy of Cheung and Lee [9] and Özkaya [10] was studied on different structures. The former sets the target service level at the warehouse and the penalty cost at the retailers; meanwhile the latter sets the target service level only at the retailers. The $(Q, S, T)$ policy is a hybrid continuous and periodic replenishments. An order is placed at the warehouse either when the cumulative demands over all retailers reach $Q$ units or when at least one demand arrives in $T$ time units after the last ordering instance. The $(Q, S \mid T)$ policy is a periodic replenishment policy and the ordering decision arises every $T$ time units. At the decision epoch, if at least $Q$ demands have accumulated for the retailers since the last ordering instance, an order is placed at the warehouse. The $(s, S-1, S)$ policy is a continuous replenishment policy when an order is triggered and any retailer's inventory position reaches its must-order level $s$. Then other retailers in the system will be also included by this order if at least one demand arrives to each retailer. All proposed policies commonly have the retailer's order-up-to level $S$ to which the warehouse replenishes all retailers' inventories. Özkaya [10] showed comparative results among these policies without comparing to the lower bound or the best-known solution. Then, we tested Özkaya [10]'s results in the case of crossdocking system (no inventory on-hand at the warehouse) in order to develop a simple lower bound ${ }^{1}$ for comparison. The testing results showed a vast amount of cost gap between the Özkaya [10]'s best solution and the lower bound (156\% on average).

Gou et al. [11] introduced a joint replenishment policy where the warehouse takes a traditional reorder point-based stock policy and the retailers utilize the can-order $(s, c, S)$ policy. The can-order policy has the same mechanism as the $(s, S-1, S)$ policy except the can-order level $c$. When an order is triggered by a retailer, other retailers whose inventory position reaches its can-order level $c$ will be included by this order

\footnotetext{
${ }^{1}$ The simple lower bound can be determined by two steps. The first step is to find the order quantity $Q$ for all retailers by assuming that they are replenished at the same order interval. The second step is to find the reorder point $S$ responding to the target service level. Thus, the total system-wide cost can be calculated from such two decision variables.
} 
as well. Even though zero lead time was assumed in their study, they cannot provide an analytical model due to the complication. Thus, computer simulation was used instead. Their result showed that about 5 to $20 \%$ of the cost can be saved as comparing with the independent controlled policy at the retailers. Pukcarnon et al. [12] also confirmed the advantage of the can-order policy on OWNR by experimenting on the broader ranges of relevant factors. The can-order policy can save the total system-wide cost by over $30 \%$ depending on relevant factors. Nevertheless, both works did not provide a solution approach for setting the appropriate inventory policy. There are other researches on joint ordering decision conducted on different cost structures. Cross-docking systems were carried out in Özkaya [8] and Gürbüz [13]. Cetinkaya and Lee [14] studied vendor managed inventory (VMI) system without the holding cost at retailers. Axsäter and Zhang [15] developed a joint ordering policy by not concerning the shared ordering cost.

According to the existing literatures, coordinated ordering decision has been considered in various systems. It is interesting to study inventory policy setting for both warehouse and all retailers under typical cost structure containing the ordering costs and the holding costs at warehouse and all retailers. Since the can-order policy not only performs well as found in Gou et al. [11] and Pukcarnon et al. [12] but also be straightforward and appealing to one's common sense [16]. Thus, in this paper we focus on the can-order policy for OWNR. The can-order policy was first introduced by Balinfy [17], and then it was carried out by many researchers in different ways [18-24]. The can-order policy was intensively studied on the multi-item single-location inventory system. Heretofore few of previous researches focused on determining the appropriate inventory policy setting for the can-order policy in OWNR. Hence, this paper's objective is to propose a heuristic approach to determine the appropriate can-order policy in OWNR. We extend the knowledge of the can-order policy into the two-echelon inventory system in this study.

The paper is organized into six parts. Section 2 describes our problem with the relevant factors and assumptions. Section 3 explains overall methodology used in the research. Section 4 proposes a heuristic approach with preliminary analysis, concept, mathematical model and algorithm. Section 5 demonstrates the experimental results with analysis and discussion. Section 6 concludes all valuable findings and proposes ideas to extend this research on the can-order policy for OWNR in future studies.

\section{Problem Description}

The system consists of a warehouse and multiple retailers with single commodity. Let $n$ denote the number of retailers and $i$ denote the location where the warehouse is set by $i=0$ and the retailer $i \in N, N \in\{1$, $2, \ldots, n\}$. Warehouse is assigned in the first echelon called warehouse echelon, and all retailers are assigned in the second echelon called retailer echelon. Demands come from each retailer's customers defined as end customers. A warehouse and multiple retailers are cooperated as a single firm to concern the total systemwide cost under global information and centralized control. The warehouse is available to hold inventories for supplying all retailers' orders. Inventories at warehouse are fulfilled by an outside supplier whose ample stock is not considered in the problem. The warehouse distributes all required items to the retailers in a single trip without splitting lot. It is supposed that uncapacitated vehicle is available to supply all required items in the order. Multiple retailers have their own inventories to serve their customer demands. Poisson demand is assumed to represent the customer demands, denoted by $\lambda_{i}$ which is a constant mean of customer demand at the retailer $i$.

Regarding the can-order $\left(s_{i}, c_{i}, S_{i}\right)$ policy applied to our system, it has two reorder points: the mustorder level $s_{i}$ providing normal replenishment, and the can-order level $c_{i}$ making special replenishment. Special replenishment is an opportunity of a retailer's joint replenishment when other retailers reach their must-order levels. When the inventory position of any retailer drops to or below its must-order level $s_{i}$, an order is triggered to create normal replenishment. Then, other retailers in the system can also be included by this order if their inventory position is at or below its can-order level $c_{i}$; a special replenishment is occurred. All the involved retailers' inventories are fulfilled from the warehouse to their own order-up-to level $S_{i}$. Considering single commodity, the warehouse modifies the can-order policy to a traditional $\left(s_{0}, S_{0}\right)$ policy by setting its can-order level equals its must-order level. The warehouse issues an order when its inventory position reaches its must-order level $s_{0}$. Then the outside supplier will replenish the warehouse's inventory to its order-up-to level $S_{0}$. The warehouse places an order to the outside supplier if and only if retailer echelon triggers an order to the warehouse. We differentiate between order cycle at 
retailer echelon and order cycle at warehouse echelon by defining "dispatch cycle" and "replenishment cycle" for retailer echelon and warehouse echelon, respectively.

Our system considers all inventory costs at both echelons. The inventory costs are composed of 1) The holding costs at the warehouse and all retailers, 2) The major ordering costs for warehouse echelon and retailer echelon, and 3) The minor ordering costs for retailer echelon. The holding cost occurs at each location having physical stock. The total holding cost over the time period at location $i\left(H C_{i}\right)$ can be determined from the unit holding cost $\left(h_{i}\right)$ and the accumulated inventory over the time period $\left(I N V_{i}\right)$. The major ordering cost is the fixed cost occurring once an order is triggered. This cost includes administrative costs, material handling costs, and transportation costs which do not depended on the number of retailers in the order. So, the retailers in the system can share the major ordering cost together for replenishing in one round trip. The total major ordering cost over the time period at retailer echelon $\left(M J_{r}\right)$ is the retailers' major ordering cost per order $\left(K_{r}\right)$ multiplied by the number of dispatch cycle $\left(N D_{r}\right)$. Similarly, the total major ordering cost over the time period at warehouse echelon $\left(M J_{w}\right)$ is the multiplication of the warehouse' major ordering cost per order $\left(K_{w}\right)$ and the number of replenishment cycle $\left(N R_{w}\right)$. The minor ordering cost is an additional cost of each retailer when replenishing their inventories, such as additional transportation cost relating to distance or other charges. This cost depends on the number of involved retailers in that order. The total minor ordering cost over the time period $\left(M N_{r}\right)$ is accumulated from the involved retailers in each order multiplied by its minor ordering cost of retailer $i$ $\left(\kappa_{i}\right)$ over the time period. Prior works on coordinated ordering decision ignored this additional cost in spite of the fact that this additional cost directly affects the inventory policy setting [23, 24].

The concept of the can-order policy is balancing among reduced major ordering costs, varied minor ordering costs, and increased holding costs. Reduced major ordering cost occurs if a special replenishment is included in an order. On the other hand, from special replenishment there is a residual stock [23] which is a stock left above the must-order level at the order-triggered point. Then, the involved retailers have to hold more stock increasing the holding cost. Meanwhile, the minor ordering costs can be either reduced or increased depending on the order frequency at each retailer. Hence, we have to consolidate all relevant costs to determine the appropriate inventory policy setting under the total system-wide cost minimization.

It is, however, difficult to deal with the problem mainly because of the demand uncertainty, variation of retailers' order quantity, retailer's two-order point setting, and order time synchronization at all locations. In this paper we simplify the problem by assuming zero lead time. Retailers' order is instantly dispatched from the warehouse. All retailers' must-order levels are then equal to zero $\left(s_{i}=0, i \in N\right)$. The warehouse's order is also replenished from the outside supplier immediately. In this case, warehouse's must-order level is equal to -1 because the warehouse is allowed to hold zero inventory level until the next replenishment will be issued. This can help the warehouse not to keep the excessive stock waiting for the next dispatch to retailer echelon. Therefore, decision variables are $c_{i}, S_{i}$ and $S_{0}$. The notations and problem formulation are demonstrated as follows:

$n=$ Number of retailers in the system

$i=$ Index of location; the warehouse $i=0$ and the retailer $i \in N$

$T=$ The time period considered in the problem (time units)

$s_{0}=$ The must-order level at the warehouse (units); (Assign $s_{0}=-1$ from the zero-lead time assumption)

$S_{0}=$ The order-up-to level at the warehouse (units)

$s_{i}=$ The must-order level at retailer $i$ (units); (Assign $s_{i}=0$ from the zero-lead time assumption)

$c_{i}=$ The can-order level at retailer $i$ (units)

$S_{i}=$ The order-up-to level at retailer $i$ (units)

$\lambda_{i}=$ Demand rate of retailer $i$ (units/time unit)

$h_{0}=$ The unit holding cost per unit time at the warehouse (\$/unit - time unit)

$h_{i}=$ The unit holding cost per unit time at retailer $i$ (\$ $/$ unit - time unit)

$K_{w}=$ The warehouse's major ordering cost per a replenishment cycle (\$/time)

$K_{r}=$ The retailers' major ordering cost per a dispatch cycle $(\$ /$ time $)$ 
$\kappa_{i}=$ The minor ordering cost at retailer $i(\$)$

$T C\left(c_{i}, S_{i}, S_{0}\right)=$ The total system-wide cost per unit time (\$/ time unit)

$H C_{i}=$ The total holding cost at location $i$ over the time $T$ units (\$)

$M J_{r}=$ The total major ordering cost at retailer echelon over the time $T$ units (\$)

$M N_{r}=$ The total minor ordering cost at retailer echelon over the time $T$ units (\$)

$M J_{w}=$ The total major ordering cost at warehouse echelon over the time $T$ units (\$)

$I N V_{i}=$ The accumulated inventory over time period at location $i$ (unit - time unit)

$N D_{r}=$ The total number of dispatch cycle over the time $T$ units (times)

$N R_{w}=$ The total number of replenishment cycle over the time $T$ units (times)

$\delta_{(i, j)}=$ An indicator which equals 1 when retailer $i$ is included in the dispatch cycle $j$ and equals 0 otherwise

Objective function:

$$
\text { Minimize } T C\left(c_{i}, S_{i}, S_{0}\right)=\frac{\left(\sum_{i=0}^{n} H C_{i}+M J_{r}+M N_{r}+M J_{w}\right)}{T}
$$

where

$$
\begin{gathered}
H C_{i}=h_{i} \times I N V_{i} \\
M J_{r}=K_{r} \times N D_{r} \\
M N_{r}=\sum_{j=1}^{N D_{r}} \sum_{i=1}^{n} \delta_{(i, j)} \kappa_{i} \\
M J_{w}=K_{w} \times N R_{w}
\end{gathered}
$$

The objective function of the problem is to minimize the total system-wide cost per unit time. Since $s_{i}$ and $s_{0}$ can be given by the zero-lead time assumption, the total system-wide cost per unit time can be a function of only three decision variables: $c_{i}, S_{i}, S_{0}$. This enables us to simpler manipulate the problem. However, the problem remains the complications, such as demand uncertainty, variation of retailers' order quantity, and order-time synchronization at all locations.

\section{Methodology}

Dealing with the complication of our problem, the optimal solution cannot be simply derived from an analytical approach. Hence, we initially study the can-order policy on OWNR by using computer simulation. Computer simulation is an efficient approach representing the inventory process even in the complicated system. The preliminary study leads us to developing a heuristic approach. In addition, from the simulation we can determine the best-known solution used to measure the proposed heuristic approach's performance.

\subsection{Computer Simulation}

The computer algorithm representing the inventory process is illustrated in Fig. 1. The inputs for simulating the system can be divided into three groups as follows: 


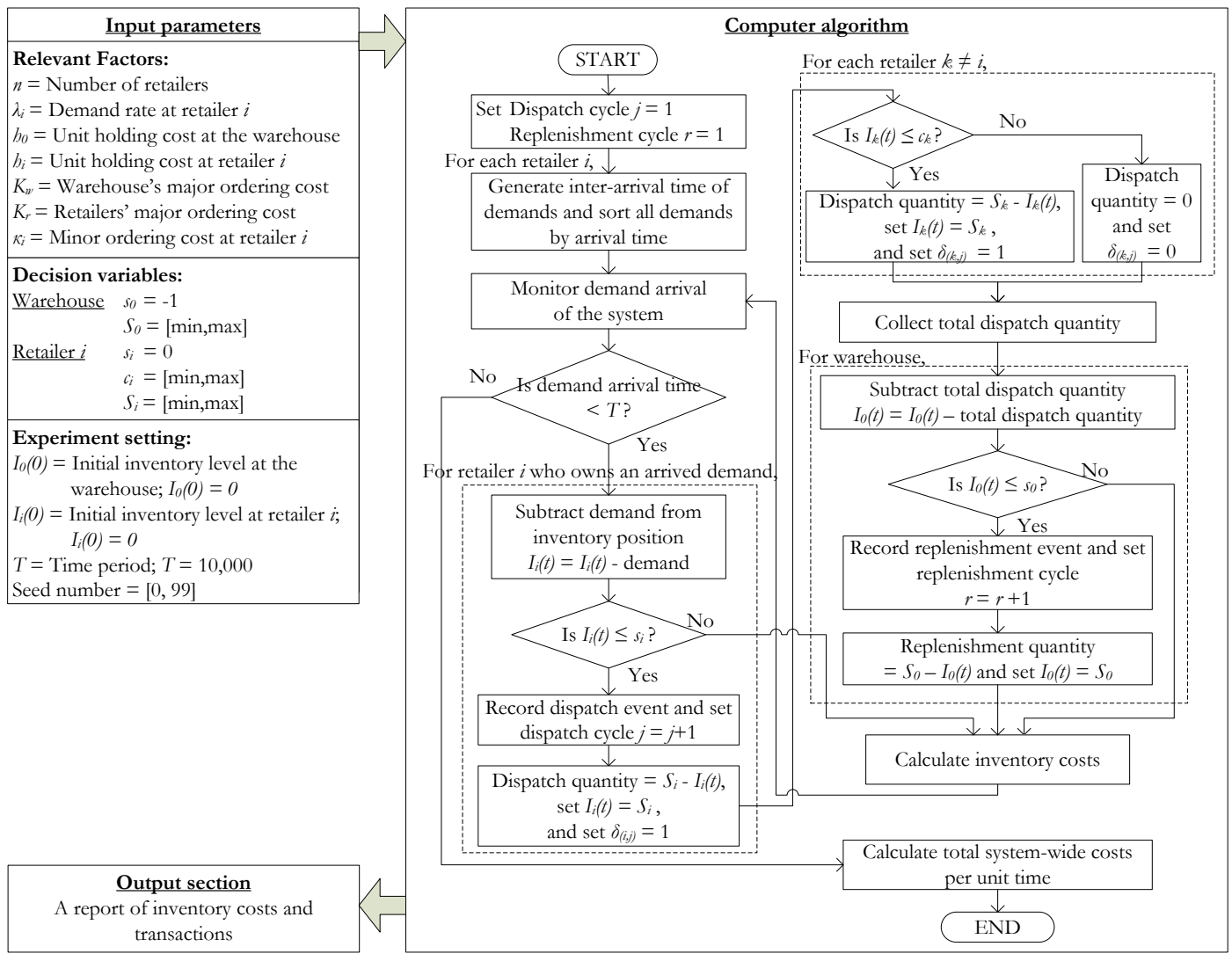

Fig. 1. The computer algorithm for simulation

1) Decision variables $\left(c_{i}, S_{i}, S_{0}\right)$ : Each variable is inputted as a range of minimum and maximum values. A combination of $\left(c_{i}, S_{i}, S_{0}\right)$ is called "solution". A solution provides a value of the total systemwide cost and its transaction (e.g. number of dispatch cycles, number of replenishment cycles).

2) Relevant factors (i.e. cost parameters, demand rates, and number of retailers): We set a combination of relevant factors to "scenario". A scenario contains different solutions. The best solution providing the minimum total system-wide cost is selected for each scenario.

3) Experiment setting: Let $I_{i}(t)$ denote the inventory level of location $i$ at time $t$. At the beginning of the running period, all locations' initial inventory levels start at zero, $I_{i}(0)=0$. We chose 10,000 running periods for our simulation, since this running period provides the steady state for the system. Additionally, various seed numbers are tested to verify the solutions since different seed numbers generate different inter-arrival time sets.

Finally, we obtain a report of the inventory costs and its transaction. In consequence, we can find the minimum total system-wide cost for each range of decision variables inputted under a given scenario.

\subsection{The Best Solution Finding}

\subsubsection{Input parameters}

First of all, we randomly select a seed number between [0, 99] to use for the first replication (i.e. a replication comes from a seed number). Decision variables are inputted as a range of minimum and maximum values. The range is dynamic depending on our setting. In the experiment, we set the width of range to be 5 units for $c_{i}$ and $S_{i}$ and 20 units for $S_{0}$. Since over 5 units of $c_{i}$ and $S_{i}$ creates multiplied combinations spending more running time. Whereas the $S_{0}$ range is larger because $S_{0}$ linearly creates combinations. The first range can be set from the initial point of $S_{0}$ and $S_{i}$ calculated by 
$S_{0}=\sqrt{2 K_{w} \sum_{i \in N} \lambda_{i} / h_{0}}$ and $S_{i}=\sqrt{2 K_{r} \lambda_{i} / h_{i}}$ due to the zero-lead time assumption and the concept of economic order quantity. For example, initial $S_{0}=45$ and initial $S_{i}=14$, the first ranges are identified as $S_{0} \in[41,60], S_{i} \in[11,15]$, and $c_{i} \in[10,14]$.

The next step is the process of moving the ranges until the solution seems to be worse continuously. The $S_{0}$ range is moved upward and downward by fixing the range at all retailers. Then, we find the $c_{i}$ and $S_{i}$ ranges at the retailer $i$ by keeping the same range of $S_{0}$ and the $c_{j}$ and $S_{j}$ ranges at the retailer $j \neq i$. $S_{0}, c_{i}$ and $S_{i}$ ranges are changed repeatedly. We select the best solution providing the minimized total system-wide cost for the first replication. After that, the validation process shown in the next part is utilized to get the typical best solution.

\subsubsection{Output validation}

The typical best solution is a representative of the best solutions from various replications. We define the typical best solution as "the best-known solution" to generally use in later sections. Since abundant combinations are run in the first replication, in this process we can reduce unnecessary ranges by starting at the best solution's range from the first replication. By this process, we can find the best solution for other replications faster. If there is an error from the first replication, cross-checking is occurred.

In the pilot testing (10 scenarios), we tested on ten random seed numbers to determine the best solution for each seed number. We found that the best-known solution appeared since the first three random seed numbers were conducted. Thus, instead of a number of the experiments we could save the computational time on five random seed numbers for determining each seed number's best solution.

Consequently, we test another four replications on different random seed numbers (after the first replication has been done previously). Most replications provide the same best solution; however, some different solutions can appear. Then, for each best solution we determine the average total system-wide cost by additional 10 random seed numbers. The best-known solution is provided by the best solution with the minimum average total system-wide cost.

\subsection{Performance Measurement}

Since this paper's objective is to propose a heuristic approach for setting the appropriate can-order policy, the best-known solution is utilized to compare with the heuristic's best solution. Heuristic's performance is measured in terms of the cost gap calculated from the following equation.

$$
\operatorname{Cost} \operatorname{Gap}(C . G .)=\frac{\left(T C^{(H R T)}-T C^{(B S)}\right) \times 100}{T C^{(B S)}}
$$

where $T C^{(H R T)}$ and $T C^{(B S)}$ are the average total system-wide cost per unit time of the heuristic approach and the average total system-wide cost per unit time of the best-known solution, respectively.

\section{Heuristic Approach}

This section demonstrates preliminary analysis which is the main findings from the computer simulation leading us to developing a heuristic approach, as well as we simplify the complicated model and propose an algorithm to determine the inventory policy setting.

\subsection{Preliminary Analysis}

In the preliminary study, the experiment was conducted for 208 scenarios (see Appendix A). Identical retailers are considered in the experiment to study the effect of relevant factors. Significant findings are demonstrated as follows: 


\subsubsection{The order-up-to level at the warehouse}

For a given $S_{0}$, we can find the solution of $\left(c_{i}, S_{i}\right)$ providing the minimum average total system-wide cost as illustrated in Fig. 2. There are two local minimum solutions located in two ranges: Range I - the solution occurs at $S_{0}=0$ and Range II - it occurs at $S_{0}>0$. For Range I, $S_{0}$ starts from zero and then increases to reach the last value before the cost line turns to a convex function. For Range II, it is defined after that last value to positive infinity. The best-known solution (global minimum solution) definitely occurs in either Range I or Range II.

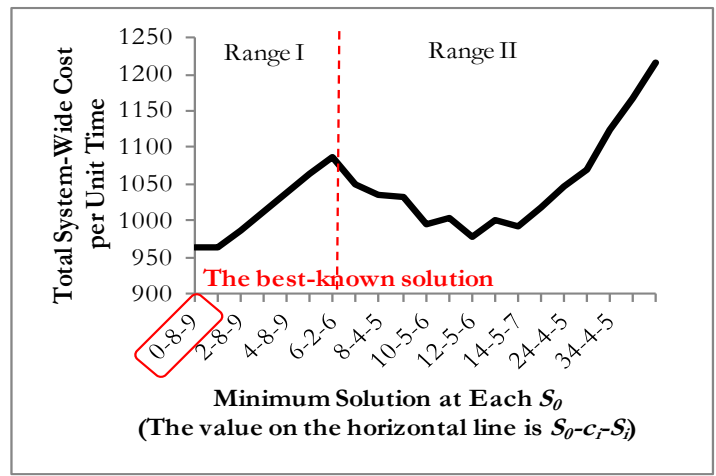

(a)

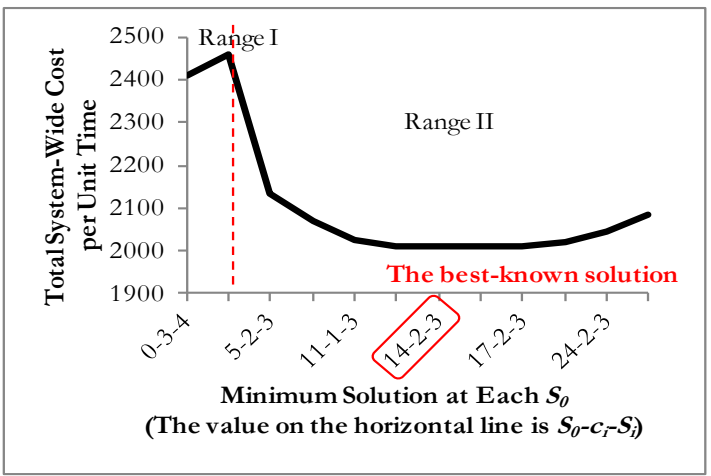

(b)

Fig. 2. Two ranges of the best-known solution: (a) the best-known solution occurred in Range I; and (b) the best-known solution occurred in Range II.

For Range I, none of holding stock at the warehouse provides the lowest total system-wide cost since the increasing $S_{0}$ creates the excessive stock. Whenever retailer echelon triggers an order all excessive stock is consumed and the warehouse's must-order level is always reached. The warehouse is replenished every dispatch cycle; therefore, it is not necessary to keep stock waiting for the next dispatch cycle. For Range II, a trade-off between the increasing holding costs and the reduced ordering costs for an increasing $S_{0}$ is occurred as found in the economic order quantity.

We can set $S_{0}=0$ for a high $h_{0} / h_{i}$ ratio, since more stock creates more inventory cost (i.e. the increased holding cost is larger than the reduced ordering cost). However, there is a possibility that the best-known solution can move from Range I to Range II when a relevant factor is changed, such as smaller $h_{0} / h_{i}$ ratio, higher $K_{w}$, or higher number of retailers since such situations affect the warehouse to hold inventories so as to reduce the frequency of replenishment.

\subsubsection{The can-order level at the retailers}

From the existing literatures, the ratio of the major ordering cost and the minor ordering cost is one of the most significant factors for the can-order policy's performance, since such ratio affects the can-order level $c_{i}$ to create a combination of retailers in an order $[23,24]$. Therefore, we consider the experiments in case of zero minor ordering cost and non-zero minor ordering cost.

Regarding the case of zero minor ordering cost (154 scenarios), a result demonstrates that for $87.66 \%$ of all scenarios (135 scenarios) the value $c_{i}^{*}=S_{i}^{*}-1$, where $c_{i}^{*}$ and $S_{i}^{*}$ denote the optimal can-order level and the optimal order-up-to level of retailer $i$. This result is consistent with the study of van Eijs [23] which showed that when $K_{r} / \kappa_{i}$ ratio is approaching infinity, then $c_{i}^{*}=S_{i}^{*}-1$ for all items. It implies that all items are jointly replenished as soon as an item triggers an order. Other items are not ordered if there has been no demand after the preceding order. This concept's purpose is to mostly reduce the ordering cost from jointly replenishing all items in the order. For other 19 scenarios occurring the best solution at $c_{i}^{*} \neq$ $S_{i}^{*}-1$, the result indicates that $T C_{\left(S_{i}-1\right)}^{*}$ is greater than $T C^{*} 0.01 \%$ on average with a standard deviation of 
$0.02 \%$ where $T C^{*}$ is the optimal average total system-wide cost and $T C_{\left(S_{i}-1\right)}^{*}$ is the minimum average total system-wide cost of the solution at $c_{i}=S_{i}-1$.

In case of non-zero minor ordering cost (54 scenarios), smaller $K_{r} / \kappa_{i}$ ratio influences a large difference between $c_{i}^{*}$ and $S_{i}^{*}$ as shown in Fig. 3(a). Since such difference can reduce the number of involved retailers in the order and dispatch quantity, but increase dispatch frequency. In the multi-item single location problem, van Eijs [23] ruled that if $K_{r} / \kappa_{i}$ ratio is less than 5, the can-order policy might not happen to be $c_{i}^{*}=S_{i}^{*}$ - 1 . Additionally, high demand rate affects a higher level gap between $c_{i}^{*}$ and $S_{i}^{*}$. Comparing $T C_{\left(S_{i}-1\right)}^{*}$ and $T C^{*}$, the result indicates that $T C_{\left(S_{i}-1\right)}^{*}$ is greater than $T C^{*}$ by $0.91 \%$ on average with a standard deviation of $1.85 \%$. Smaller $K_{r} / \kappa_{i}$ ratio increases cost gap as shown in Fig. 3(b). Setting $c_{i}$ near $S_{i}$ increases the total ordering cost because of too many retailers included in an order.

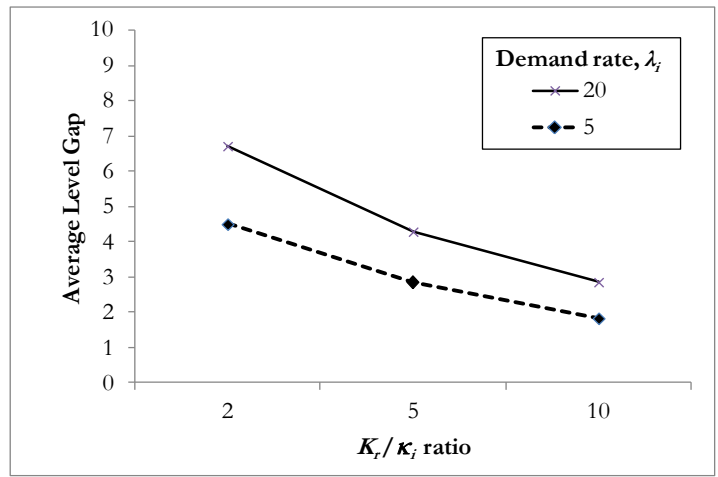

(a)

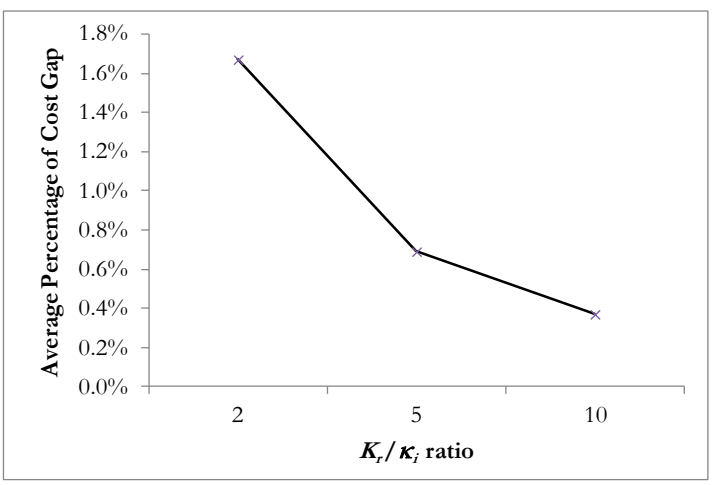

(b)

Fig. 3. The effect of $K_{r} / \kappa_{i}$ ratio on the can-order level at the retailers: (a) Average level gap between $c_{i}^{*}$ and $S_{i}^{*}$, and (b) Average percentage of cost gap between $T C_{\left(S_{i}-1\right)}^{*}$ and $T C^{*}$.

\subsubsection{The order-up-to level at the retailers}

When we fix the inventory policy at the warehouse, the average total system-wide cost at retailer $i$ is a convex (unimodal) function of $S_{i}$ as shown in Fig. 4. Figure 4(a) and Figure 4(b) illustrate different scenarios but provide the same pattern. The convex function occurs from a trade-off between the increasing holding costs and the reduced ordering costs for an increasing $S_{i}$, then the economic order quantity is determined. 


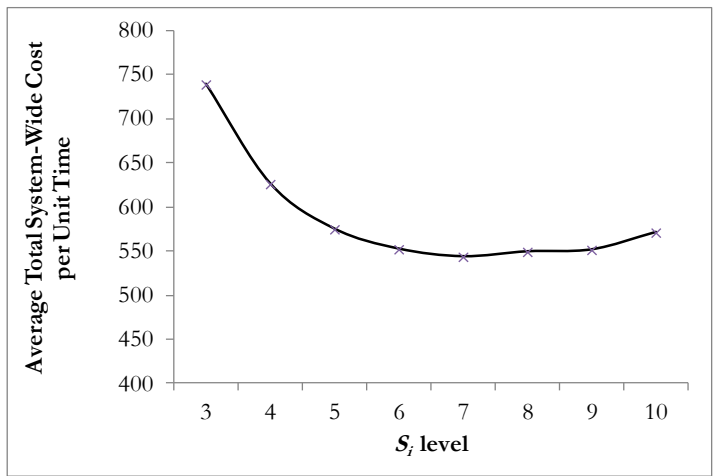

(a)

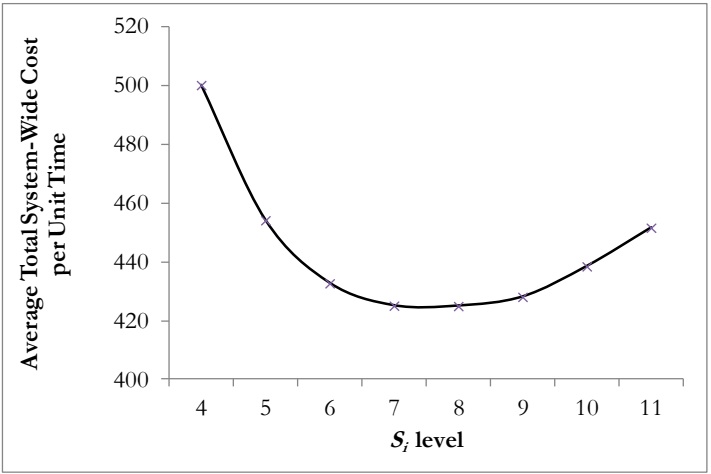

(b)

Fig. 4. Convex function of $S_{i}$ on given $S_{0}$ : (a) Scenario at $K_{w}=100, K_{r}=50, \kappa_{i}=0, h_{0}=2.5, h_{i}=25$, $\lambda_{i}=20, n=2, S_{0}=48$; and (b) Scenario at $K_{w}=100, K_{r}=50, \kappa_{i}=25, h_{0}=2, h_{i}=10, \lambda_{i}=5$, $n=4, S_{0}=27$.

As the above results, we can simplify the mathematical model by using the can-order level $c_{i}=S_{i}-1$ since small average cost gap between $T C_{\left(S_{i}-1\right)}^{*}$ and $T C^{*}$ is occurred. Additionally, a convex function of $S_{i}$ enables us to develop a heuristic approach at ease with one-dimensional search.

\subsection{Mathematical Model}

Our purpose of developing a heuristic approach is to provide an appropriate inventory policy $\left(c_{i}, S_{i}, S_{0}\right)$. The total system-wide cost of mathematical model is able to be approximated as long as the acceptable solution is provided. This can reduce the complexity of our model. Hence, relating to the preliminary analysis our mathematical model utilizes the can-order level at $c_{i}=S_{i}-1$. Then, there exists only two decision variables $\left(S_{i}, S_{0}\right)$ concerned in the mathematical model. Van Eijs [23] developed exact equations by using $c_{i}=S_{i}-1$ for non-identical items on single location. His model used the exact probability of the special replenishment, unlike other models assuming Poisson distributions. It performed very well when the $K_{r} / \kappa_{i}$ ratio is more than 5 . Therefore, we adapt his work into our consideration.

Based on van Eijs [23], we can calculate the inventory cost at the retailer echelon close to the exact value. However, determination of inventory cost at warehouse is another difficult part. The warehouse's inventory level is consumed by an uncertain lot-sizing order from retailer echelon. From preliminary testing, we determine the expected dispatch quantity at retailer echelon by using the exact model of van Eijs [23]. We found that the expected dispatch quantity per dispatch cycle is always equal to the cumulative demand from all retailers. Thus, we simplify this part by assuming that the warehouse's inventory level is consumed continuously following the total Poisson demand cumulated from all retailers, $\lambda_{0}=\sum_{i \in N} \lambda_{i}$. By this assumption, warehouse echelon and retailer echelon are independent to find the minimum inventory costs at each echelon. Even though the assumption provides the approximate warehouse's inventory cost higher than the warehouse's actual inventory cost, we compensate the approximate value by utilizing the minimum inventory cost at retailer echelon.

The cost model can be formulated for a given $\left(S_{i}, S_{0}\right)$ policy. It follows that,

$$
T C\left(S_{i}, S_{0}\right)=\frac{\left.K_{r}+\sum_{i \in N}\left\{\left(1-\Phi\left(S_{i}\right)\right) \times \kappa_{i}\right)+E\left[H_{i}\right]\right\}}{E[D T]}+\frac{K_{w}+E\left[H_{0}\right]}{E[R T]}
$$

$T C\left(S_{i}, S_{0}\right) \quad=$ The long-run average total system-wide cost per unit time $(\$ /$ time unit $)$

$\Phi\left(S_{i}\right) \quad=$ The probability that no demand arrives for retailer $i$ during a dispatch cycle

$E\left[H_{i}\right] \quad=$ The expected holding cost of retailer $i$ during a dispatch cycle $(\$)$ 


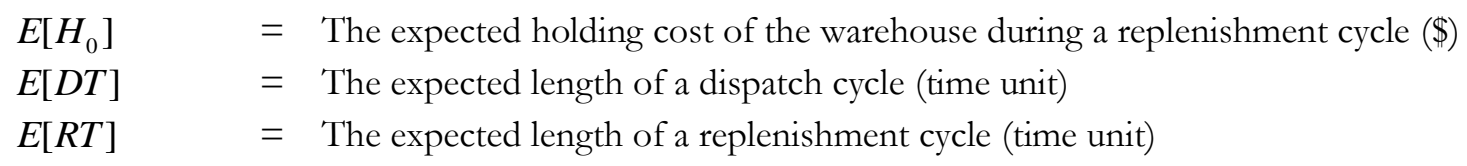

According to the equation (7), we consider the probability that at least one demand arrives for retailer $i$ during a dispatch cycle to be consistent with the value $c_{i}=S_{i}-1$. Such probability affects the occurrence of the minor ordering cost.

\section{Retailer Echelon}

The model is developed according to the independent Poisson process of demands for individual retailers, so inter-arrival times of demands are exponentially distributed. Suppose a dispatch cycle starts at time 0 . We define the following variables according to stochastic process:

$D T_{i}=$ Time until retailer $i$ triggers an order to the warehouse (time unit)

$D T=$ Time until any retailer triggers an order to the warehouse; $D T=\min \left(D T_{i}\right)$ (time unit)

$f_{i}(t)=$ Probability density function of $D T_{i}$

$F_{i}(t)=$ Distribution function of $D T_{i}$

$f(t) \quad=$ Probability density function of $D T$

$F(t)=$ Distribution function of $D T$

Retailer $i$ will trigger an order if the total demand for retailer $i$ from time 0 equals $S_{i}$. Thus, according to the exponential distribution of inter-arrival times of demands, $D T_{i}$ follows Erlang distribution with parameters $\lambda_{i}$ and $S_{i}$. The value of $f_{i}(t)$ and $F_{i}(t)$ are determined by the general formula of Erlang distribution [25]. Then, the probability density function and distribution function of $D T$ can be calculated by

$$
\begin{gathered}
f(t)=\sum_{i \in N} f_{i}(t) \prod_{j \neq i}\left(1-F_{i}(t)\right) \\
F(t)=1-\prod_{i \in N}\left(1-F_{i}(t)\right)
\end{gathered}
$$

Thus, the expected length of a dispatch cycle is

$$
E[D T]=\int_{t=0}^{\infty} t f(t) d t=\int_{t=0}^{\infty}(1-F(t)) d t=\int_{t=0}^{\infty} \prod_{i \in N}\left(1-F_{i}(t)\right) d t
$$

The expected holding cost of retailer $i$ during a dispatch cycle is associated with the retailer's inventory on hand at the beginning and at the end of the dispatch cycle. At the beginning of the cycle, setting $c_{i}=S_{i}$ - 1 makes all retailers' inventory on hand equal $S_{i}$. At the end of the cycle, the inventory on hand depends on the residual stock level, which is a stock above the must-order level when an order is triggered. Thus, we define $\Phi_{i}(x)$ as the probability that at time $D T$ the residual stock of retailer $i$ equals $x$. There are two cases for determining $\Phi_{i}(x)$. The first case is when the residual stock level of retailer $i$ is equal to zero; only retailer $i$ triggers an order. The second case is when the residual stock level of retailer $i$ is positive. So, an order is triggered by retailer $j \neq i$. Thus, the value of $\Phi_{i}(x)$ can be calculated by the following expressions: 


$$
\Phi_{i}(x)=\left\{\begin{array}{cc}
\int_{t=0}^{\infty} f_{i}(t) \prod_{j \neq i}\left(1-F_{j}(t)\right) d t & \text { if } x=0, \\
\int_{t=0}^{\infty} \operatorname{Pois}\left(\lambda_{i}, S_{i}-x\right) f^{(-i)}(t) d t & \text { if } 0<x \leq S_{i} \\
\operatorname{Pois}\left(\lambda_{i}, m\right)=\frac{\lambda_{i}^{m} e^{-\lambda_{i}}}{m !} & \\
f^{(-i)}(t)=\sum_{j \neq i} f_{j}(t) \prod_{k \neq j, i}\left(1-F_{k}(t)\right)
\end{array}\right.
$$

where $\operatorname{Pois}\left(\lambda_{i}, m\right)$ is the probability density function of Poisson demand at retailer $i$, and $f^{(-i)}(t)$ is the probability density function that at time $t$ any retailer $j \neq i$ triggers an order. Thus, $\Phi\left(S_{i}\right)$ illustrated in equation (7) can be calculated by using equation (11) as well.

The expected holding cost of retailer $i$ during a dispatch cycle is then given by

$$
E\left[H_{i}\right]=\sum_{x=0}^{S_{i}}\left\{\Phi(x) \int_{t=0}^{\infty} \frac{h_{i}\left(S_{i}+x\right) t}{2} f(t) d t\right\}
$$

According to the equation (10) and (14), we transform the expression to determine the expected holding cost of retailer $i$ per unit time instead. Thus,

$$
\frac{E\left[H_{i}\right]}{E[D T]}=\sum_{x=0}^{S_{i}}\left\{\Phi(x) \frac{h_{i}\left(S_{i}+x\right)}{2}\right\}
$$

\section{Warehouse Echelon}

To simplify this part, we assume that the warehouse's inventory level is consumed continuously by all retailers' Poisson demands with rate $\lambda_{0}$. Inter-arrival times of demands are exponentially distributed, and then the distribution of time until warehouse triggers an order to an outside supplier is Erlang, similar to the retailer echelon. Let $R T$ denote the time until warehouse triggers an order to an outside supplier. The warehouse will trigger an order if the total demand from time 0 equals $S_{0}$, so the distribution of $R T$ is Erlang with parameters $\lambda_{0}$ and $S_{0}$. The expected length of a replenishment cycle is the mean of Erlang distribution. Thus, $E[R T]=S_{0} / \lambda_{0}$.

In case of holding inventory at the warehouse, the expected holding cost of the warehouse during a replenishment cycle is estimated following the continuous demands from the retailer echelon. Then, we can determine the expected holding cost of the warehouse per unit time by $\frac{E\left[H_{0}\right]}{E[R T]}=\frac{h_{0} S_{0}}{2}$. According to the expression at the warehouse, we can find the optimal order-up-to level at the warehouse $S_{0}^{*}$ from the derivative of the cost function with respect to $S_{0}$. We found that $S_{0}^{*}$ can be easily calculated from $E O Q$ formula. Then, $S_{0}^{*}=\sqrt{2 K_{w} \lambda_{0} / h_{0}}$

Consequently, we can figure out the long-run average total system-wide cost per unit time for a given $\left(S_{i}, S_{0}\right)$ policy. Then, the next section will demonstrate the algorithm of heuristic approach to determine the appropriate decision variables by using the cost model.

\subsection{The Algorithm of Heuristic Approach}

With regard to the preliminary analysis and the mathematical model, the following analyses demonstrate our concept for developing the heuristic approach. 
1) According to two local minimum solutions located into two ranges, we can identify the value of $S_{0}$ to $S_{0}=0$ for Range I and $S_{0}=\sqrt{2 K_{w} \lambda_{0} / h_{0}}$ for Range II.

2) To develop an initial solution at retailer echelon by assuming $c_{i}=S_{i}-1$, we can use deterministic model to find economical joint ordering time when every retailers is replenished in an order.

3) Fixing inventory policy at retailer $j \neq i$ and at the warehouse, the total inventory cost at retailer $i$ is a convex function of $S_{i}$. We can find the local minimum $T C\left(S_{i}, S_{0}\right)$ at the given $S_{j \neq i}$ and $S_{0}$. Therefore, the decomposition technique and iterative procedure can be applied to break multiple locations into a single location and to recurrently find the minimum solution as far as the best solution has been found. Both techniques have been intensively used in JRP [19, 22-24, 26-28].

4) Since the total inventory cost at retailer $i$ is a unimodal function under one-dimensional unconstrained problem. We apply the line search called "golden section search" which is a simple and efficient method for finding the extremum of a unimodal function [23, 29, 30]. The golden section search is suitable for the case of non-derivative function, like our model, by successively narrowing the range of search space until the desired accuracy in the minimum value of the objective function is achieved. A golden ratio, which is a constant reduction factor for the size of the interval, is utilized to maintain the successive range of dynamic triples of points (i.e. upper point, middle point, and lower point). Advantageously, each successive range we only want to perform one new function evaluation. From this technique, we can determine the optimal $S_{i}^{*}$ for the given $S_{j \neq i}$ and $S_{0}$ and save computational time. The golden section search was verified to efficiently use with the can-order policy in van Eijs [23]'s work.

Hence, the heuristic approach is outlined in the following algorithm illustrated in Fig. 5.

In step 1 - determination of the initial solution $S_{i}$, we calculate the joint dispatching time $\left(T_{d}\right)$ by deterministic model according to the following expression:

$$
T_{d}=\sqrt{\frac{2\left(K_{w}+\sum_{i \in N} \kappa_{i}\right)}{\sum_{i \in N} \lambda_{i} h_{i}}}
$$

Then, the initial $S_{i}$ for retailer $i$ is determined by adapting Love [16]'s method. It is selecting $S_{i}$ which provides the minimum gap between two probabilities: 1) the probability that the demand for retailer $i$ during time $T_{d}$ is less than or equal to such $S_{i}$ and 2) the probability that an order is triggered by any retailer (i.e. including normal replenishment and special replenishment). Thus,

$$
S_{i}=\left\{\begin{aligned}
m & \text { if }\left\{\operatorname{Pois}\left(\lambda_{i} T_{d}, m+1\right)-\left(\frac{n}{n+1}\right)\right\} \geq\left\{\left(\frac{n}{n+1}\right)-\operatorname{Pois}\left(\lambda_{i} T_{d}, m\right)\right\} \\
m+1 \quad \text { Otherwise } & \}
\end{aligned}\right\}
$$

The initial $S_{i}$ from equation (17) is closer to the optimal solution than $S_{i}$ obtained from $S_{i}=\lambda_{i} T_{d}$. 


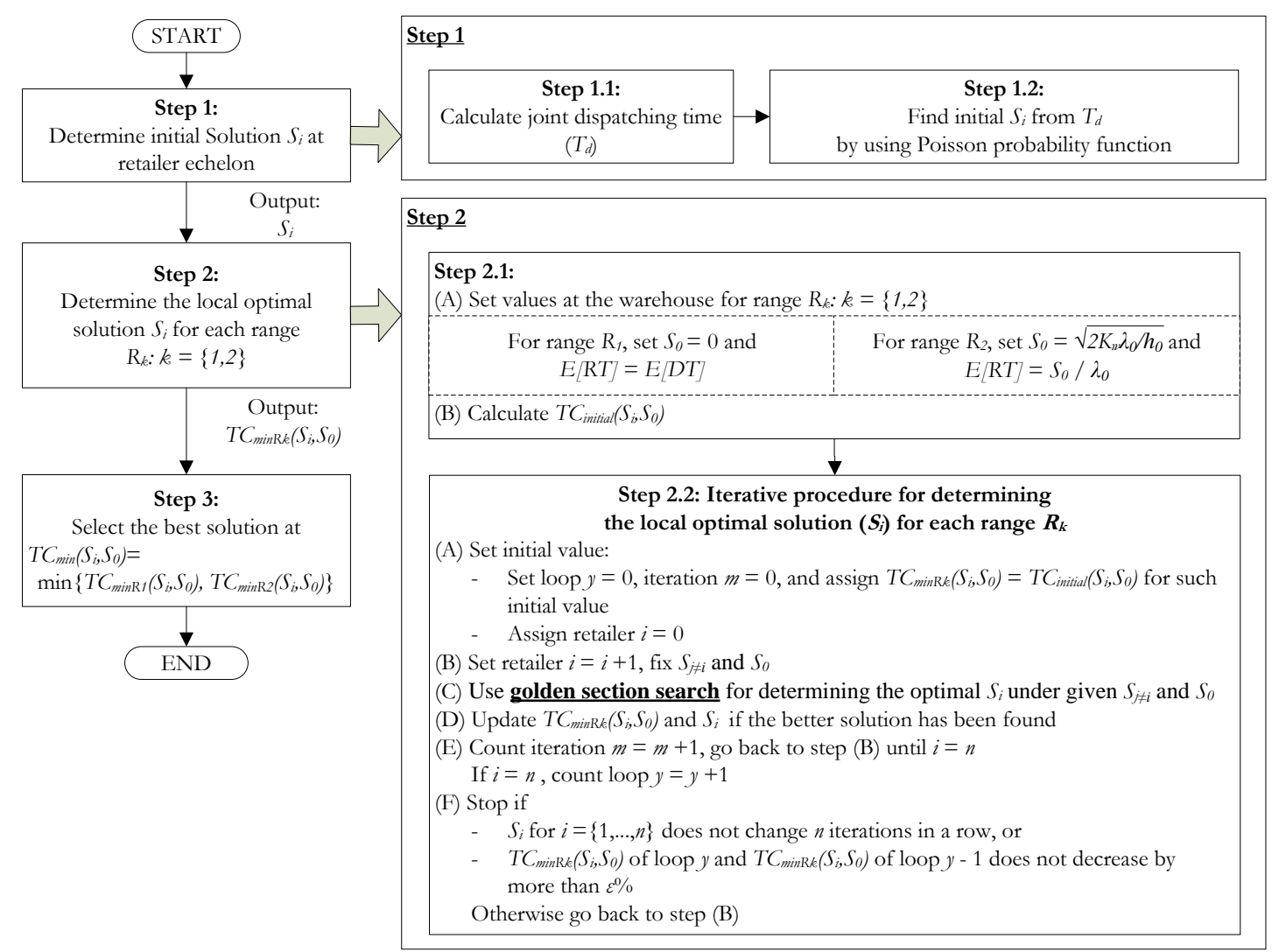

Fig. 5. The algorithm of heuristic approach.

Step 2 is the most important procedure for the heuristic in order to determine the optimal $S_{i}$ for each range of range $R_{1}$ and $R_{2}$ (note that for range $R_{1}$, the local optimal solution occurs at $S_{0}=0$ and $E[R T]=E[D T]$, and for range $R_{2}$, it occurs at $S_{0}=\sqrt{2 K_{w} \lambda_{0} / h_{0}}$ and $\left.E[R T]=S_{0} / \lambda_{0}\right)$. We use $S_{0}$ and the initial $S_{i}$ from step 1 to calculate the initial long-run average total system-wide cost per unit time, $T C_{\text {initial }}\left(S_{i}, S_{0}\right)$. The next step (2.2) is an iterative procedure containing steps (A) to (F). For each iteration, a golden section search is carried out for retailer $i$ : vary $S_{i}$ and fix $S_{j \neq i}$ given from the previous iteration. $T C\left(S_{i}, S_{0}\right)$ is an objective function for golden section search. The iterative process terminates as soon as every $S_{i}$ does not change $n$ iterations in a row, or the minimum long-run average total system-wide cost per unit time, $T C_{\min R k}\left(S_{i}, S_{0}\right)$, from the current loop does not decrease from the previous loop by more than $\varepsilon \%$ (i.e. when all retailers have been run, one loop is counted). From step 2, we obtain the local minimum cost $T C_{\min R k}\left(S_{i}, S_{0}\right)$ for $k \in\{1,2\}$.

Lastly, the comparison of $T C_{\min R k}\left(S_{i}, S_{0}\right)$ for $k \in\{1,2\}$ is carried out in step 3. The minimum long-run average total system-wide cost per unit time is equal to $\min \left\{T C_{\min R 1}\left(S_{i}, S_{0}\right), T C_{\min R 2}\left(S_{i}, S_{0}\right)\right\}$.

\section{Experimental Results}

The heuristic approach has been tested on various scenarios. The experiments on identical retailers are analyzed, specifically in the case of zero minor ordering cost and non-zero minor ordering cost. Since both cases affects the can-order policy at given $c_{i}=S_{i}-1$ on different results as shown in the preliminary analysis. In addition, the experiment on non-identical retailers is also conducted to measure the heuristic's performance on the dissimilar situation. 


\subsection{Identical Retailers}

\subsubsection{Zero minor ordering cost}

According to three relevant factors (i.e. cost parameters, demand rates, and number of retailers), they are designed to examine the heuristic's performance under 154 scenarios (see Appendix A). Table 1 shows some numerical examples relating to the best-known solution of the system and the best solution from the heuristic approach. We found that the performance of heuristic approach depends on all relevant factors. It provides an average cost gap at $1.05 \%$ with standard deviation of $1.11 \%$ over various scenarios. Our approach performs well for high number of retailers, high $K_{w} / K_{r}$ ratio, and high $h_{0} / h_{i}$ ratio.

Let $S_{i}^{(B S)}$ and $S_{0}^{(B S)}$ denote the best-known order-up-to level at retailer $i$ and at the warehouse determined from the computer simulation. Let $S_{i}^{(H R T)}$ and $S_{0}^{(H R T)}$ denote the best order-up-to level at retailer $i$ and at the warehouse and they are calculated by the heuristic approach. Theoretically, a large number of retailers increases the joint replenishment opportunity from special replenishment, this can reduce $S_{i}$. Thus, a higher number of retailers reduces $S_{i}^{(B S)}$ to be closer to $S_{i}^{(H R T)}$ and also increases $S_{0}^{(B S)}$ to be closer to $S_{0}^{(H R T)}$. Therefore, the cost gap can reduce. For higher $K_{w} / K_{r}$ ratio, $S_{i}$ and $S_{0}$ are affected in a similar pattern.

Regarding the $h_{0} / h_{i}$ ratio, higher ratio influences the warehouse's stock equal to zero. Consequently, the inventory cost at retailer echelon becomes the main part of the system. Our mathematical model provides cost expression at retailer echelon near the exact value and heuristic approach can determine the minimum solution at retailer echelon. Then, the heuristic approach provides the (near) best-known solution.

Table 3. Numerical examples for comparison of the best-know solution and the heuristic's best solution under identical retailers without minor ordering cost.

\begin{tabular}{c|c|c|c|c|c|c|c|c|c|c}
\hline \multirow{2}{*}{ Instance } & \multicolumn{9}{|c|}{ Relevant factors } & \multicolumn{2}{c}{ Best-known Solution (BS) } & \multicolumn{2}{c}{ Heuristic Approach } \\
\cline { 2 - 11 } & $K_{w}$ & $K_{r}$ & $h_{0}$ & $h_{i}$ & $\lambda_{i}$ & $n$ & $S_{0}, c_{i}, S_{i}$ & $T C^{(B S)}$ & $S_{0}, c_{i}, S_{i}$ & $C . G$. \\
\hline 1 & 100 & 50 & 20 & 100 & 20 & 2 & $13,3,4$ & $1,280.75$ & $20,3,4$ & $0.52 \%$ \\
\hline 2 & 100 & 50 & 40 & 100 & 20 & 2 & $0,5,6$ & $1,420.94$ & $0,5,6$ & $0.00 \%$ \\
\hline 3 & 100 & 50 & 2 & 10 & 20 & 2 & $45,9,12$ & 359.73 & $63,10,11$ & $2.03 \%$ \\
\hline 4 & 100 & 50 & 4 & 10 & 20 & 2 & $25,12,13$ & 392.37 & $0,18,19$ & $1.88 \%$ \\
\hline 5 & 100 & 10 & 2 & 10 & 20 & 2 & $58,4,5$ & 244.97 & $63,4,5$ & $0.05 \%$ \\
\hline 6 & 100 & 90 & 2 & 10 & 20 & 2 & $31,15,16$ & 424.21 & $63,14,15$ & $4.34 \%$ \\
\hline 7 & 125 & 50 & 2 & 10 & 20 & 2 & $49,12,13$ & 376.43 & $71,10,11$ & $2.50 \%$ \\
\hline 8 & 250 & 50 & 2 & 10 & 20 & 2 & $86,11,12$ & 436.63 & $100,10,11$ & $0.98 \%$ \\
\hline 9 & 100 & 50 & 2 & 10 & 10 & 4 & $42,6,7$ & 427.16 & $63,5,6$ & $0.36 \%$ \\
\hline 10 & 100 & 50 & 2 & 10 & 10 & 8 & $78,4,5$ & 697.16 & $89,4,5$ & $0.11 \%$ \\
\hline 11 & 100 & 50 & 2 & 10 & 10 & 12 & $93,4,5$ & 932.60 & $110,4,5$ & $0.15 \%$ \\
\hline 12 & 100 & 50 & 2 & 10 & 20 & 4 & $79,8,9$ & 576.83 & $89,8,9$ & $1.03 \%$ \\
\hline 13 & 100 & 50 & 2 & 10 & 20 & 8 & $100,6,7$ & 925.98 & $126,6,7$ & $0.29 \%$ \\
\hline 14 & 100 & 50 & 2 & 10 & 20 & 12 & $142,5,6$ & $1,230.39$ & $155,5,6$ & $0.17 \%$ \\
\hline
\end{tabular}

\subsubsection{Non-zero minor ordering cost}

Although the can-order level is not necessary to be equal to $S_{i}-1$ when there is a minor ordering cost, our heuristic approach can be applied into this problem in some situations. To identify such a situation, we tested on 54 scenarios (see Appendix A) by mainly varying the minor ordering cost $\kappa_{i}$. The value of $K_{r} / \kappa_{i}$ ratio are identified following van Eijs's work [23]. The experimental results are depicted in Fig. 6. 


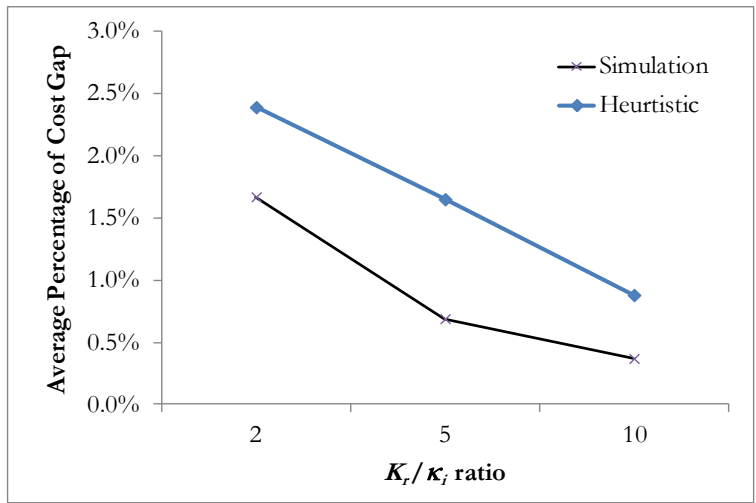

(a)

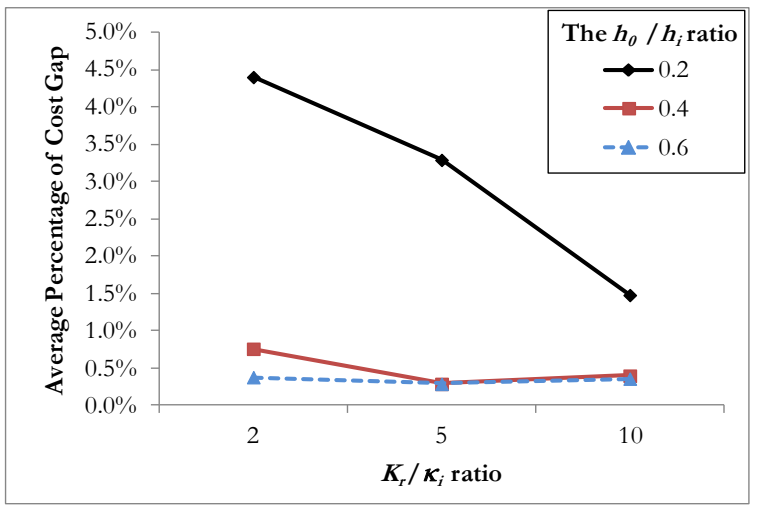

(b)

Fig. 6. The effect of $K_{r} / \kappa_{i}$ ratio on the can-order level at the retailers: (a) Heuristic's performance and simulation's performance when fixing $c_{i}=S_{i}$ - 1 , and (b) The effect of $h_{0} / h_{i}$ ratio.

We found that the heuristic approach provides an average cost gap at $1.64 \%$ with standard deviation of $2.03 \%$ over various scenarios. Heuristic's performance is associated with two reasons. Firstly, our heuristic assumes $c_{i}=S_{i}$-1. As shown in Fig. 6(a). Relating to computer simulation, we compare the best-known solution with the best solution fixing $c_{i}=S_{i}$ - 1. Average percentage of cost gap provides in simulation's line. A smaller $K_{r} / \kappa_{i}$ ratio provides a larger cost gap in simulation's line, consequently our heuristic also performs in the same way. Secondly, the inventory cost at the warehouse is approximate. Cost gap of the heuristic's line is also added from the simulation's line.

Considering the $h_{0} / h_{i}$ ratio, a higher ratio $\left(h_{0} / h_{i}\right.$ is 0.4 and 0.6$)$ influences the warehouse's stock equal to zero. Then, the heuristic approach provides the (near) best-known solution. On the other hand, a higher cost gap at the lower $h_{0} / h_{i}$ ratio comes from an approximate inventory cost at the warehouse, especially for a small demand rate and high number of retailers by the reason that our heuristic gets $S_{0}=0$ whereas the best-known solution is $S_{0}>0$. The difference of solution creates a larger cost gap.

\subsection{Non-Identical Retailers}

To extend the experiment on non-identical retailers, we aim at studying the can-order policy on the retailers' different demand rates because in reality we frequently encounter such situation. In addition, non-identical demands can create the different discount opportunities from the shared ordering cost. Hence, it is interesting to investigate and this inquiry has not been studied in the existing literatures. We tested on tworetailer scenarios and three-retailer scenarios (see Appendix B). Figure 7 depicts the cost gap from our heuristic approach, as compared to the best-known solutions.

The heuristic approach provides an average cost gap at $2.18 \%$ with standard deviation of $0.82 \%$ for two-retailer scenarios, and an average cost gap at $1.80 \%$ with standard deviation of $0.51 \%$ for three-retailer scenarios. At small demand rate ratio the heuristic approach performs well because order cycle of each retailer is not quite different. So, the retailers' ordering cost can be more shared with the balancing holding costs. However, at a higher demand rate ratio heuristic's performance does not depend on the different demand rates (i.e. there is no trend of the cost gap following the demand rate ratio). 


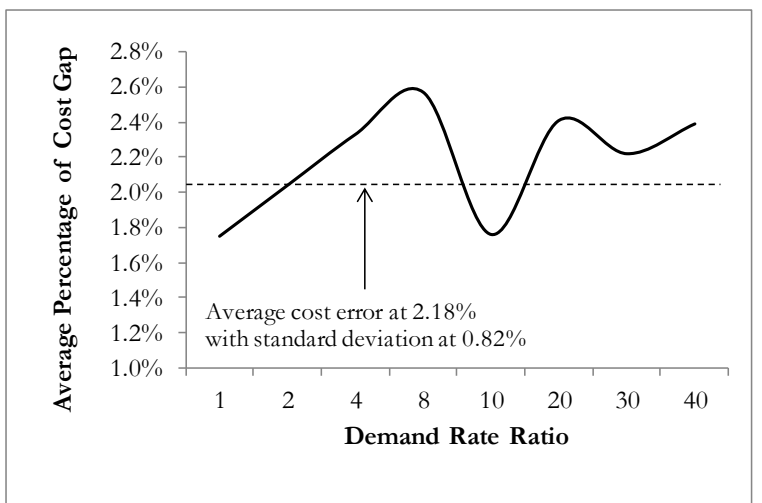

(a)

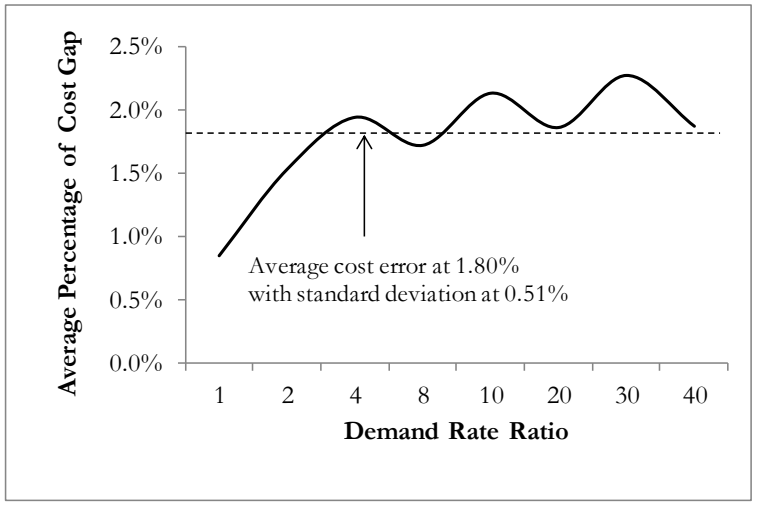

(b)

Fig. 7. Heuristic's performance under non-identical retailers: (a) Two-retailer scenarios, and

(b) Three-retailer scenarios.

As the experimental results in various scenarios, the heuristic approach provides the best solutions at a small average cost gap comparing to the best-known solution. Moreover, the heuristic approach's computational time can be saved from the reduced search space as comparing to the computer simulation's computational time. Since the golden section search can save the computational time by reducing the searching points. It is a satisfactory approach to use for the can-order policy setting under OWNR.

\section{Conclusions}

This paper proposed a heuristic approach for determining on appropriate can-order policy into onewarehouse n-retailer inventory system. Dealing with the complication of our problem, computer simulation was employed to explore insights into the can-order policy and to determine the best-known solution. The insights led us to developing the mathematical model and the algorithm of the heuristic approach.

The main findings showed from computer simulation that the average total system-wide cost is a unimodal function of the retailer's order-up-to level $S_{i}$, when given $S_{j \neq i}$ and $S_{0}$ are fixed. Decomposition technique and iterative procedure can be applied to break multiple locations into a single location and to successively find the minimum as far as the best solution has been found. Since our mathematical model is a non-derivative function, we utilized golden section search for finding the minimum of a unimodal function. This can save our computational time to find the appropriate inventory policy setting.

The heuristic approach under simplified mathematical model and fixed $c_{i}=S_{i}-1$ performs very well, especially in case of high $K_{r} / \kappa_{i}$ ratio. Overall, the experiments tested on the wide range of data provided the cost gap of heuristic approach of less than $2 \%$ on average. With satisfactory computational time and small cost gap, the heuristic approach is well worth using for the can-order policy setting under the onewarehouse n-retailer inventory system.

In this research, two main contributions were gained. Firstly, the zero lead time assumption can be interpreted and applied in the situation when the ratio of lead time to order cycle duration is very small. Secondly, our study can be used as the base case when we extend into non-zero lead time. Furthermore, multiple items should be concerned to totally utilize the can-order policy on the warehouse. 


\section{Appendix A: Preliminary Experiment}

In the preliminary study, the experiment is designed on the identical retailers. The following table shows 208 scenarios experimented in sequence. The asterisk $\left(^{*}\right)$ in the table means that parameter is varied.

Table A1. Numerical input for preliminary experiment under the identical retailers

\begin{tabular}{c|c|c|c|c|c|c|c|c|c}
\hline \multirow{2}{*}{ Scenario No. } & $K_{w}$ & $K_{r}$ & $\kappa_{i}$ & $h_{0}$ & $h_{i}$ & $h_{0} / h_{i}$ & $\lambda_{i}$ & $n$ & Varied Parameters \\
\cline { 2 - 7 } & 1) Relationship between $h_{0}$ and $h_{i}(80$ scenarios $)$ \\
\hline $1-50$ & 100 & 50 & 0 & $*$ & $*$ & $*$ & 20 & 2 & $h_{i} \in\{10,25,50,100,250\} ; h_{0} / h_{i} \in\{0.1,0.2, \ldots, 1\}$ \\
\hline $51-80$ & 100 & 50 & 0 & $*$ & $*$ & $*$ & 20 & 2 & $\begin{array}{l}h_{i} \in\{0.1,0.5,1,2.5,5\} ; \\
h_{0} / h_{i} \in\{0.1,0.3,0.5,0.7,0.9,1\}\end{array}$ \\
\hline
\end{tabular}

2) Relationship between $h_{0}, h_{i}$ and $K_{r}$ (20 scenarios)

\begin{tabular}{c|c|c|c|c|c|c|c|c|c}
\hline $81-92$ & 100 & $*$ & 0 & $*$ & 25 & $*$ & 20 & 2 & $K_{r} \in\{10,90\} ; h_{0} / h_{i} \in\{0.1,0.3,0.5,0.7,0.9,1\}$ \\
\hline $93-100$ & 100 & $*$ & 0 & $*$ & 10 & $*$ & 20 & 2 & $K_{r} \in\{10,90\} ; h_{0} / h_{i} \in\{0.2,0.4,0.6,0.8\}$ \\
\hline
\end{tabular}

3) Relationship between $h_{0}, h_{i}$ and $K_{w}$ (20 scenarios)

\begin{tabular}{c|c|c|c|c|c|c|c|c|l}
\hline $101-112$ & $*$ & 50 & 0 & $*$ & 25 & $*$ & 20 & 2 & $K_{w} \in\{75,200\} ; h_{0} / h_{i} \in\{0.1,0.3,0.5,0.7,0.9,1\}$ \\
\hline $113-120$ & $*$ & 50 & 0 & $*$ & 10 & $*$ & 20 & 2 & $K_{w} \in\{125,250\} ; h_{0} / h_{i} \in\{0.2,0.4,0.6,0.8\}$ \\
\hline
\end{tabular}

4) Relationship between $h_{0}, h_{i}$, and $K_{w} / K_{r}$ (14 scenarios)

\begin{tabular}{|c|c|c|c|c|c|c|c|c|c|}
\hline $121-134$ & $*$ & 50 & 0 & * & * & 0.5 & 20 & 2 & $K_{w} / K_{r} \in\{1.5,3,4,5,10,100,1500\} ; h_{i} \in\{1,25\}$ \\
\hline \multicolumn{10}{|c|}{ 5) Relationship between $h_{0}, h_{i}$, and $\lambda_{i}$ (10 scenarios) } \\
\hline $135-142$ & 100 & 50 & 0 & $*$ & 25 & 0.5 & $*$ & 2 & $\lambda_{i} \in\{0.5,1,3,5,10,40,100,500\}$ \\
\hline $143-144$ & 100 & 50 & 0 & * & 10 & 0.2 & $*$ & 2 & $\lambda_{i} \in\{0.5,10\}$ \\
\hline
\end{tabular}

6) Relationship between $h_{0}, h_{i}, \lambda_{i}$ and $n$ (10 scenarios)

\begin{tabular}{c|c|c|c|c|c|c|c|c|l}
\hline $145-148$ & 100 & 50 & 0 & $*$ & 25 & 0.5 & 20 & $*$ & $n \in\{4,8,12,20\}$ \\
\hline $149-154$ & 100 & 50 & 0 & $*$ & 10 & 0.2 & $*$ & $*$ & $\lambda_{i} \in\{0.5,10\} ; n \in\{4,8,12\}$ \\
\hline
\end{tabular}

7) The effect of $\kappa_{i}$ (54 scenarios)

\begin{tabular}{l|l|l|l|l|l|l|l|l|l}
\hline $155-208$ & 100 & 50 & $*$ & $*$ & 10 & $*$ & $*$ & $*$ & $\begin{array}{l}\kappa_{i} \in\{5,10,25\} ; \lambda_{i} \in\{0.5,20\} ; \\
h_{0} / h_{i} \in\{0.2,0.4,0.6\} ; n \in\{2,4,8\}\end{array}$ \\
\hline
\end{tabular}




\section{Appendix B: The Experiment on Non-Identical Retailers}

The following table shows 45 scenarios on two-retailer problem and three-retailer problem. All scenarios set identical cost components by $K_{w}=100, K_{r}=50, \kappa_{i}=0, h_{0}=2$, and $h_{i}=10$.

Table B1. Numerical input for the experiment on non-identical retailers:

\begin{tabular}{|c|c|c|c|c|c|c|c|c|c|c|c|c|c|c|}
\hline \multirow{2}{*}{ 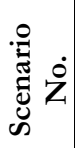 } & \multicolumn{4}{|c|}{ Demand Rate } & \multirow{2}{*}{ 逜 } & \multicolumn{4}{|c|}{ Demand Rate } & \multirow{2}{*}{ 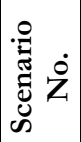 } & \multicolumn{4}{|c|}{ Demand Rate } \\
\hline & $\lambda_{1}$ & $\lambda_{2}$ & $\lambda_{3}$ & $\begin{array}{l}\text { Demand } \\
\text { Rate Ratio }\end{array}$ & & $\lambda_{1}$ & $\lambda_{2}$ & $\lambda_{3}$ & $\begin{array}{c}\text { Demand } \\
\text { Rate Ratio }\end{array}$ & & $\lambda_{1}$ & $\lambda_{2}$ & $\lambda_{3}$ & $\begin{array}{l}\text { Demand } \\
\text { Rate Ratio }\end{array}$ \\
\hline 1 & 20 & 20 & - & \multirow{3}{*}{1} & 16 & 20 & 1 & - & \multirow{3}{*}{20} & 31 & 20 & 0.67 & 0.67 & 30 \\
\hline 2 & 10 & 10 & - & & 17 & 10 & 0.5 & - & & 32 & 20 & 0.5 & 0.5 & 40 \\
\hline 3 & 40 & 40 & - & & 18 & 40 & 2 & - & & 33 & 20 & 20 & 10 & 2 \\
\hline 4 & 20 & 10 & - & \multirow{3}{*}{2} & 19 & 20 & 0.67 & - & \multirow{3}{*}{30} & 34 & 20 & 20 & 5 & 4 \\
\hline 5 & 10 & 5 & - & & 20 & 10 & 0.33 & - & & 35 & 20 & 20 & 2.5 & 8 \\
\hline 6 & 40 & 20 & - & & 21 & 40 & 1.33 & - & & 36 & 20 & 20 & 2 & 10 \\
\hline 7 & 20 & 5 & - & \multirow{3}{*}{4} & 22 & 20 & 0.5 & - & \multirow{3}{*}{40} & 37 & 20 & 20 & 1 & 20 \\
\hline 8 & 10 & 2.5 & - & & 23 & 10 & 0.25 & - & & 38 & 20 & 20 & 0.67 & 30 \\
\hline 9 & 40 & 10 & - & & 24 & 40 & 1 & - & & 39 & 20 & 20 & 0.5 & 40 \\
\hline 10 & 20 & 2.5 & - & \multirow{3}{*}{8} & 25 & 20 & 20 & 20 & 1 & 40 & 20 & 10 & 5 & 2,4 \\
\hline 11 & 10 & 1.25 & - & & 26 & 20 & 10 & 10 & 2 & 41 & 20 & 10 & 0.5 & $2,20,40$ \\
\hline 12 & 40 & 5 & - & & 27 & 20 & 5 & 5 & 4 & 42 & 40 & 20 & 10 & 2,4 \\
\hline 13 & 20 & 2 & - & \multirow{3}{*}{10} & 28 & 20 & 2.5 & 2.5 & 8 & 43 & 40 & 20 & 1 & $2,20,40$ \\
\hline 14 & 10 & 1 & - & & 29 & 20 & 2 & 2 & 10 & 44 & 20 & 2 & 0.5 & $4,10,40$ \\
\hline 15 & 40 & 4 & - & & 30 & 20 & 1 & 1 & 20 & 45 & 40 & 4 & 1 & $4,10,40$ \\
\hline
\end{tabular}

\section{Acknowledgement}

The Scholarship from the Graduate School, Chulalongkorn University to commemorate the 72nd anniversary of his Majesty King Bhumibala Aduladeja is gratefully acknowledged. The authors would like thank the anonymous associate editor, and referees for their helpful and valuable comments. A portion of the work was presented at 17th International Conference on Industrial Engineering Theory, Applications and Practice (IJIE 2013).

\section{References}

[1] B. D. Williams and T. Tokar, "A review of inventory management research in major logistics journals: Themes and future directions," the International Journal of Logistics Management, vol. 19, no. 2, pp. 212-232, 2008.

[2] P. Kelle, H. Schneider, S. Wiley-Patton, and J. Woosley, "Healthcare supply chain management," in Inventory Management: Non-classical views. M. Y. Jaber, Ed., Baco Raton: CRC Press, 2009, pp. 99-128.

[3] K. Arshinder, A. Kanda, and S. G. Deshmukh, "A Review on supply chain coordination: Coordination mechanisms, managing uncertainty and research directions," Supply Chain Coordination under Uncertainty. Berlin Heidelberg: Springer, 2011, pp. 39-82.

[4] S. Axsäter, S. C. Graves, and A. G. d. Kok, "Supply chain operations: Serial and distribution inventory systems," Handbooks in Operations Research and Management Science, Elsevier, 2003, pp. 525-559.

[5] Q. Wang, T.-M. Choi, and T. C. E. Cheng, "Control policies for multi-echelon inventory systems with stochastic demand," in Supply Chain Coordination under Uncertainty. Berlin Heidelberg: Springer, 2011, pp. 83-108.

[6] H. Schneider, D. B. Rnks, and P. Kelle, "Power approximations for a two-echelon inventory system using service levels," Production and Operations Management, vol. 4, no. 4, pp. 381-400, 1995. 
[7] S. C. Graves, "On the deterministic demand multi-product single-machine lot scheduling problem," Management Science, vol. 25, no. 3, pp. 276-280, March, 1979, 1979.

[8] B. Y. Özkaya, Ü. Gürler, and E. Berk, "The stochastic joint replenishment problem: A new policy, analysis, and insights," Naval Research Logistics (NRL), vol. 53, no. 6, pp. 525-546, 2006.

[9] K. L. Cheung and H. L. Lee, "The inventory benefit of shipment coordination and stock rebalancing in a supply chain," Management Science, vol. 48, no. 2, pp. 300-306, 2002.

[10] B. Y. Özkaya, "Stochastic joint replenishment problem: A new policy and analysis for single location and two echelon inventory systems," Ph.D. dissertation, Industrial Engineering, Bilkent University, Turkey, 2005.

[11] Q. Gou, L. Liang, C. Xu, and Y. Zha, "A modified joint inventory policy for VMI systems," International Journal of Information Technology \& Decision Making (IJITDM), vol. 07, no. 02, pp. 225-240, 2008.

[12] V. Pukcarnon, P. Chaovalitwongse, and N. Phumchusri, "An application of the can-order policy for one-warehouse n-retailer inventory system," in 17th International Conference on Industrial Engineering: Theory, Applications and Practice, Pusan National University, Busan, Korea, 2013, pp. 378-395.

[13] M. C.. Gürbüz, K. Moinzadeh, and Y.-P. Zhou, "Coordinated replenishment strategies in inventory/distribution systems," Management Science, vol. 53, no. 2, pp. 293-307, Feb., 2007.

[14] S. Cetinkaya and C.-Y. Lee, "Stock replenishment and shipment scheduling for vendor-managed inventory systems," Management Science, vol. 46, no. 2, pp. 217-232, Feb., 2000.

[15] S. Axsäter and W.-F. Zhang, "A joint replenishment policy for multi-echelon inventory control," International Journal of Production Economics, vol. 59, pp. 243-250, 1999.

[16] S. F. Love, "Coordinated replenishment of multiple items," Inventory Control. Auckland: McGraw-Hill, 1979, pp. 111-141.

[17] J. L. Balintfy, "On a basic class of multi-item inventory problems," Management Science, vol. 10, no. 2, pp. 287-297, January, 1964, 1964.

[18] E. A. Silver, "Some characteristics of a special joint-order inventory model," Operations Research, vol. 13, no. 2, pp. 319-322, Mar.-Apr., 1965.

[19] E. A. Silver, "A control system for coordinated inventory replenishment," International Journal of Production Research, vol. 12, no. 6, pp. 647-671, 1974.

[20] R. M. Thompstonej and E. A. Silver, "A coordinated inventory control system for compound Poisson demand and zero lead time," International Journal of Production Research, vol. 13, no. 6, pp. 581-602, 1975.

[21] E. A. Silver, "Establishing reorder points in the (S,c,s) coordinated control system under compound Poisson demand," International Journal of Production Research, vol. 19, no. 6, pp. 743-750, 1981.

[22] A. Federgruen, H. Groenevelt, and H. C. Tijms, "Coordinated replenishments in a multi-item inventory system with compound Poisson demands," Management Science, vol. 30, no. 3, pp. 344-357, March, 1984.

[23] M. J. G. van Eijs, "On the determination of the control parameters of the optimal can-order policy," Zeitschrift für Operations Research, vol. 39, no. 3, pp. 289-304, 1994.

[24] P. Melchiors, "Calculating can-order policies for the joint replenishment problem by the compensation approach," European Journal of Operational Research, vol. 141, no. 3, pp. 587-595, 2002.

[25] C. Forbes, M. Evans, N. Hastings, and B. Peacock, "Erlang distribution," Statistical Distributions, 4th ed. John Wiley \& Sons, Inc., 2010, pp. 84-85.

[26] Y.-S. Zheng, "Optimal control policy for stochastic inventory systems with Markovian discount opportunities," Operations Research, vol. 42, no. 4, pp. 721-738, 1994.

[27] N. Dellaert and E. van de Poel, "Global inventory control in an academic hospital," International Journal of Production Economics, vol. 46-47, pp. 277-284, 1996.

[28] H. Schultz and S. G. Johansen, "Can-order policies for coordinated inventory replenishment with Erlang distributed times between ordering," European Journal of Operational Research, vol. 113, no. 1, pp. 30-41, 1999.

[29] M. Evans, N. A. J. Hastings, and J. B. Peacock, Statistical Distributions, 3rd ed. Wiley, 2000.

[30] W. H. Press, S. A. Teukolsky, W. T. Vetterling, and B. P. Flannery, Numerical Recipes: The art of scientific computing, 3rd ed. Cambridge University Press, 2007. 